\title{
In vivo imaging of malaria parasites in the murine liver
}

\author{
Sabine Thiberge ${ }^{1}$, Samantha Blazquez ${ }^{1}$, Patricia Baldacci ${ }^{1}$, Olivier Renaud ${ }^{2}$, Spencer Shorte ${ }^{2}$, Robert Ménard ${ }^{1}$ \\ \& Rogerio Amino ${ }^{1,3}$
}

\begin{abstract}
${ }^{1}$ Unité de Biologie et Génétique du Paludisme, ${ }^{2}$ Plate-forme d’Imagerie Dynamique, Institut Pasteur, 25-28 rue du Dr Roux, 75724 Paris cedex 15, France. ${ }^{3}$ Present address: Departamento de Bioquimica, Universidade Federal de Sao Paulo, Rua Três de Maio 100, 04044-020, Sao Paulo, SP, Brazil. Correspondence should be addressed to R.M. (rmenard@pasteur.fr) or R.A. (roti@ecb.epm.br).
\end{abstract}

Published online 19 July 2007; doi:10.1038/nprot.2007.257

\begin{abstract}
The form of the malaria parasite inoculated by the mosquito, called the sporozoite, transforms inside the host liver into thousands of a new form of the parasite, called the merozoite, which infects erythrocytes. We present here a protocol to visualize in vivo the behavior of Plasmodium berghei parasites in the hepatic tissue of the murine host. The use of GFP-expressing parasites and a high-speed spinning disk confocal microscope allows for the acquisition of four-dimensional images, which provide a time lapse view of parasite displacement and development in tissue volumes. These data can be analyzed to give information on the early events of sporozoite penetration of the hepatic tissue, that is, sporozoite gliding in the liver sinusoids, crossing the sinusoidal barrier, gliding in the parenchyma and traversal of hepatocytes, and invasion of a final hepatocyte, as well as the terminal events of merosome and merozoite release from infected hepatocytes. Combined with the use of mice expressing fluorescent cell types or cell markers, the system will provide useful information not only on the primary infection process, but also on parasite interactions with the host immune cells in the liver.
\end{abstract}

\section{INTRODUCTION}

Malaria infection occurs when a mosquito containing Plasmodium parasites takes a blood meal, during which the infective form or sporozoite is injected into the dermis of the host ${ }^{1-5}$. At least some of the sporozoites deposited in the dermis eventually enter the blood circulation and reach the hepatic tissue ${ }^{5}$, where they invade hepatocytes and transform into the so-called liver or exo-erythrocytic stage. A liver stage, which originates from a single sporozoite and matures over a period of 2-3 days in rodent systems, leads to the emergence of tens of thousands of merozoites, the parasite stage that infects erythrocytes ${ }^{6}$. Invasion of erythrocytes then leads to the symptoms and complications of the disease.

The liver phase of malaria infection comprises numerous host-parasite interactions, and our understanding of this part of the parasite life cycle is still incomplete. Most of our views on how the parasite goes into and out of the hepatic tissue come from transmission electron microscopy approaches, whose significance is frequently limited by the small number of events reported, or from in vitro studies, which have their own limitations ${ }^{7-9}$. In recent years, imaging technology has been transferred from physics laboratories to the biological domain with impressive efficiency, which has revolutionized the way in vivo questions can be tackled $^{10,11}$. There are presently various confocal microscopy techniques available that permit the acquisition of four-dimensional (4D) data, that is, in space and time. Among these techniques are (i) laser scanning confocal microscopy, which may cause high cell damage in living cells owing to the generation of oxygen radicals and is limited by the slow speed of data acquisition, (ii) multi-photon microscopy ${ }^{12}$, which offers the advantage of deep tissue penetration with minimal cell damage but is also limited by the slow speed of data acquisition, and (iii) highspeed spinning disk confocal microscopy ${ }^{13}$, which causes little cell damage and allows high-speed data acquisition. Combined with the construction of recombinant rodent malaria parasites ${ }^{14-16}$ that express the GFP at the pre-erythrocytic stages, these developments now permit in vivo analysis of Plasmodium behavior in rodent systems.

Two in vivo imaging studies of Plasmodium parasites in the liver of rodents have been published so far. The first ${ }^{17}$ used confocal laser scanning and conventional epifluorescence microscopy to address the way sporozoites cross the liver sinusoid endothelial barrier from the sinusoidal lumen to the parenchyma. This study concluded that Kupffer cells, the resident macrophages of the liver, act as gateways in the process. The second ${ }^{18}$ used high-speed spinning disk confocal microscopy to focus on the way hepatic merozoites gain access to the bloodstream. It revealed the existence of merosomes, which are host cell-derived buds containing numerous parasites that detach from the infected mother cell to reach the blood circulation.

We present here a protocol for studying Plasmodium parasites in the murine host hepatic tissue. The sporozoite is a highly motile cell that can glide inside host tissues at the speed of up to $2-4 \mu \mathrm{m} \mathrm{s}^{-1}$. The use of high-speed spinning disk confocal microscopy, which permits high-speed data acquisition, limits cell damage that occurs during prolonged sample illumination. This technology is suited for tracking fast-moving objects and can image up to $80 \mu \mathrm{m}$ into the tissue, which is sufficient to observe the morphology of the liver parenchyma.

The protocol indicates how to record and analyze $4 \mathrm{D}$ data of fluorescent parasites during the early steps of sporozoite penetration through the hepatic parenchyma as well as during the late steps of merozoite release in the sinusoids. It should be useful for further analysis of sporozoite infection of the host liver, about which major issues remain unresolved. For example, it is still unclear whether or not sporozoites can cross the liver sinusoidal endothelial barrier via a paracellular or a transendothelial route. The fine biology of parasite maturation in vivo is also largely unexplored, and the 
recent discovery of the merosomes suggests that other complex and unexpected cell biological events may occur during liver stage maturation and merozoite release. Finally, the protocol could be adapted for visualizing the immune response of the host, and how protection proceeds in immunized individuals.

\section{Note about the procedure}

For the imaging steps, we give the concept for each point, so users can easily adapt the protocol to the equipment available to them. In some cases, we give information specific to our equipment; this is preceded by "For our equipment."

\section{MATERIALS}

REAGENTS

-18-22 days post-infection Anopheles stephensi Sda500 mosquitoes, infected with $P$. berghei

-P. berghei NK65 clone, PbFluspo, expressing GFP under the control of the cs promoter (stage-specific fluorescence at the oocyst, sporozoite and early liver stages $)^{14}$

-P. berghei ANKA clone, WT: GFP, expressing GFP under the control of the hsp70 promoter ${ }^{19}$, or $P$. berghei ANKA clone, $\mathrm{PbGFP}_{\mathrm{CON}}$, expressing GFP under the control of the ef $-1 \alpha$ promoter ${ }^{15}$; the parasites are fluorescent at all stages

- Mice: 4- to 7-week-old C57BL/6J@Rj (Elevage Janvier). Other murine backgrounds are acceptable; however, the liver morphology of C57BL/6 mice has the best structure for microscopy studies. Rats can be used, but as they are bigger animals, a higher number of sporozoites are needed for infection, so young animals should be preferred ! CAUTION All experiments involving rodents must conform to national and institutional regulations.

- Absolute ethanol (Merck, ref. 1.00983.1011) diluted to 70\% (vol/vol) in distilled water

$\cdot 1 \times$ PBS Dulbecco (Invitrogen, ref. 14040091)

- Bovine serum albumin (BSA) (Sigma) to verify gliding motility of sporozoites

-Anesthetic solution (see REAGENT SETUP): pentobarbital (CEVA) or a mixture comprising ketamine (Imalgene 1000, Merial) and xylazine

(2\% Rompun, Bayer)

- Superglue 3 (Henkel)

- Physiological serum and cotton pads

- Bovine serum albumin conjugated to Alexa Fluor 647 (Molecular ProbesInvitrogen)

EQUIPMENT

- Syringe-driven $0.22 \mu \mathrm{m}$ PES filter unit (Millipore)

- Disposable piston pellet for microtubes (Fisher Bioblock Scientific)

- Hemocytometer (CML)

$\cdot 1 \mathrm{ml}$ insulin syringes with integrated needle (Omnican $0.30 \times 12 \mathrm{~mm}$, B BRAUN)

- Microsurgery eye scissors (World Precision Instruments)

- Microsurgery straight iris scissors (World Precision Instruments)

- Microsurgery curved iris forceps (World Precision Instruments)

- Coverslips (Micro cover glass $24 \times 60 \mathrm{~mm}$; Eric Scientific Company)

- Aspirator for collecting mosquitoes

- Stereozoom microscope with epifluorescence and GFP filter for dissecting mosquitoes (Zeiss Stemi 2000-C, Carl Zeiss) with fiber-optic lighting (Schott KL1500LCD)

- Epifluorescence microscope equipped with a GFP filter for parasite counting

- Low-temperature cautery (World Precision Instruments) to cauterize any

blood vessels cut during surgery

- Epifluorescence microscope (Axiovert 200, Carl Zeiss) equipped with a high-speed spinning disk confocal system (see EQUIPMENT SETUP). This protocol can be modified to use other fluorescence microscopy techniques - Pekin-Elmer Ultraview ERS Image Suite software
.25× Plan-NeoFluar NA 0.8 multi-immersion corrected objective (Carl Zeiss) for the spinning disk system. Higher magnification objectives can be used depending on what is to be observed

- Homeothermic blanket system for animal heating (Harvard Apparatus, http://www.harvardapparatus.com/webapp/wcs/stores/servlet/ category_11051_10001_37612_-1_HAI_Categories_N_37611_37610_)

- Handmade aluminum platform or cover from rectangular cell culture dish to hold the mouse on the motorized microscope stage

- ImageJ software (see EQUIPMENT SETUP)

REAGENT SETUP

Anesthetic solution Use either a pentobarbital (in general, this anesthetic is less likely to induce hyperventilation) or ketamine/xylazine mix. Pentobarbital should be used at a concentration of $50 \mathrm{mg}$ per kg body weight. Inject i.p. $100 \mu \mathrm{l}$ of a solution appropriately diluted. For the ketamine/xylazine mix, mix two volumes of Imalgene 1000 (ketamine) with one volume of $2 \%$ Rompun (xylazine) and five volumes of $1 \times$ PBS. Inject i.p. $50 \mu \mathrm{l}$ of this solution per $10 \mathrm{~g}$ of body weight (ketamine: $125 \mathrm{mg}$ per kg body weight; xylazine: $12.5 \mathrm{mg}$ per kg body weight).

BSA-Alexa Fluor 647 Prepare a solution at $10 \mathrm{mg} \mathrm{m}^{-1}$ in sterile-filtered PBS and aliquot protected from light and store at $-20^{\circ} \mathrm{C}$.

EQUIPMENT SETUP

High-speed spinning disk confocal microscopy for in vivo imaging The minimal equipment needed consists of a fluorescence microscope, equipped with an objective with a long working distance (for high penetration into the tissue) and a high-speed charge-coupled device camera. However, to avoid photobleaching and cell damage, the use of multi-photon confocal microscopy or spinning-disk confocal microscopy is recommended.

We use the Ultraview ERS spinning disk from Perkin-Elmer (http://las. perkinelmer.com/content/livecellimaging/) equipped with an $\mathrm{Ar} / \mathrm{Kr}$ laser (excitation at three wavelengths: 488,568 and $647 \mathrm{~nm}$ ) mounted on an inverted Axiovert 200 microscope with Optovar (1.6× magnification) option (Carl Zeiss) linked to a high-speed charge-coupled device camera (Orca ER camera, Hamamatsu, Japan). Note that cameras with a higher sensitivity and frame per second value are now commercially available. The $z$-position is controlled by an objective piezo-drive System (Piezosystem Jena) via the Perkin-Elmer pilot software.

Microscope platform To be able to place the animal correctly on the microscope stage, we had a made-to-measure aluminum platform $(160 \times 110 \times$ $2 \mathrm{~mm}$ ) with a round central opening (21 mm diameter). The cover of a rectangular Petri dish for cell culture can also be used if the making of the aluminum platform is not possible. A circular or rectangular hole must be made in the center that is slightly smaller than the size of the cover glass that will be used.

Image J software Download from http://rsb.info.nih.gov/ij and install. From the Plugins menu, install the "MultiTracker Plugin" (http://rsb.info.nih.gov/ij/ plugins/multitracker.html). In the case of the Ultraview ERS Image suite software, one can use the Perkin-Elmer 4D-image series converter Plugin written to run in ImageJ, which is freely available at http://www.embl-heidelberg.de/eamnet/html/ pe_plugin.html (Marchand, M. and Shorte, S.L., PFID, Institut Pasteur)

\section{PROCEDURE}

\section{Infection of mosquitoes}

1) Infect starved $A$. stephensi female mosquitoes (200 per cage) by allowing them to feed on mice bearing the desired fluorescent parasite clone.

2| Keep infected mosquitoes in the standard way as described in ref. 20 for 18-22 days post-infection. Briefly, place a $10 \%$ (wt/vol) sugar solution and a 'laying' dish in the mosquito cages. Keep the A. stephensi mosquitoes in dedicated rooms or incubators at $21{ }^{\circ} \mathrm{C}$ and $70 \%$ humidity. Replace the sugar regularly during the 3-week period. Remove the 'laying' dish after 2 weeks. The imaging experiments are performed with sporozoites isolated from the salivary glands of mosquitoes 18-22 days 
post-infection, when mosquitoes typically contain between 10,000 and 30,000 sporozoites per salivary gland and when sporozoites have maximum infectivity ${ }^{20}$.

3| At 18-22 days post-infection, collect the female mosquitoes with an aspirator tube,'anesthetize' the collected insects by blowing them into a $15 \mathrm{ml}$ Falcon tube on ice, and carefully transfer the cold-anesthetized insects onto a Petri dish containing $70 \%$ ethanol. Transfer the mosquitoes to another Petri dish containing $1 \times$ PBS.

4| Place the mosquitoes on a glass slide and examine them using a stereomicroscope with epifluorescence (GFP filter). Identify mosquitoes that display GFP fluorescence in the salivary glands, and dissect these as described in Steps 5-10 (further details of the dissection can be found in ref. 20).

\section{Dissection of mosquitoes with infected salivary glands}

5| Dissect out salivary glands under the stereomicroscope using two insulin needles. Place one needle on the thorax and with the other pull the head away from the body. Free the salivary glands from the thorax and remove any debris from around the glands.

$\triangle$ CRITICAL STEP During dissection, maintain mosquitoes in a humid condition with a few drops of $1 \times$ PBS to avoid dehydration under the stereomicroscope.

6| Recover the salivary glands and place in a low adhesion $1.5 \mathrm{ml}$ microcentrifuge tube containing a small volume (around $10 \mu \mathrm{l})$ of $1 \times$ PBS on ice.

7| Repeat Steps 5 and 6 for about 25 mosquitoes. The number of mosquitoes to be dissected depends on the rate of infection, as an injection of $1 \times 10^{6}$ sporozoites is optimal. In general, a good infection should yield approximately 40,000-50,000 sporozoites per female. However, the number of sporozoites per female can be known only after counting as described in Steps 8 and 9.

$\triangle$ CRITICAL STEP Once dissected, maintain salivary glands on ice.

- PAUSE POINT Sporozoites in uncrushed glands are viable for up to $4 \mathrm{~h}$ on ice.

8| Gently crush the salivary glands with a disposable piston pellet for microtubes to release the sporozoites. Take $1 \mu$ of the suspension and dilute at least ten times in a $1 \times$ PBS solution containing $5 \%$ BSA.

9| Place $10 \mu \mathrm{l}$ of the dilution in a hemocytometer, and using an epifluorescence microscope with a GFP filter, count the number of sporozoites. The total volume of the 81 squares of the hemocytometer listed in the equipment is $1 \mu \mathrm{l}$. To obtain the total number of sporozoites collected, the number of sporozoites counted must be multiplied by the dilution (at least $10 \times$ ) and by the total volume $(10 \mu \mathrm{l})$. It is also important to verify the quality of sporozoite gliding motility; the presence of BSA in the diluting medium will allow the sporozoites to glide (to move in circles). The number of sporozoites able to complete a full circle of movement should be noted.

? TROUBLESHOOTING

\section{Preparing the samples}

10| The parasite can be visualized at early time points as sporozoites (A) or at later time points as hepatic forms also known as exo-erythrocytic forms (EEFs) (B). Follow the appropriate option below:

\section{(A) Sporozoites in the liver (motility and liver invasion)}

(i) Resuspend the sporozoite suspension at a concentration of $\sim 1 \times 10^{6}$ parasites per $10 \mu \mathrm{l}$ of filtered PBS and store on ice while continuing with the next steps.

\section{(B) Hepatic forms in the liver (merosomes)}

(i) Resuspend the sporozoite suspension at a concentration of $1 \times 10^{6}$ parasites per $100 \mu$ l of filtered PBS and store on ice.

(ii) Vasodilate the mouse tail vein, either by soaking the tail in warm water $\left(40^{\circ} \mathrm{C}\right)$ for 5 min or by heating under an infrared lamp.

(iii) Inject the sporozoite suspension i.v. into the mouse tail vein.

(iv) Leave the mouse for $45 \mathrm{~h}$, then continue with the next step.

\section{Starting up the equipment}

11 Turn on the necessary devices, verify that they are all working (homeothermic heater blanket system, imaging system) and install the required objective.

12| Open windows necessary for image acquisition (microscope control, laser control, camera control). Select the options necessary to acquire images with green and far-red fluorescence. The optimal conditions are a short exposure time, permitting the rapid acquisition of $z$-stacks over time (for our equipment; Supplementary Fig. 1 online). 

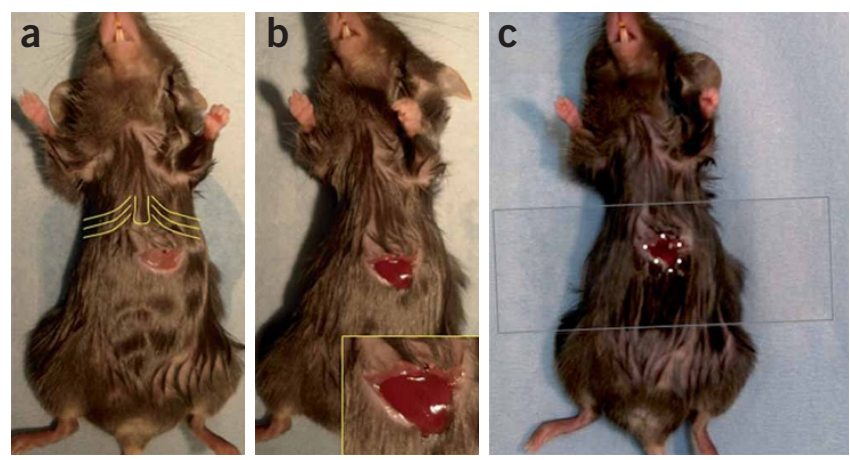

Figure 1 | Animal preparation for liver imaging. This surgical procedure leads to the exposition of the left liver lobe, facilitating imaging of this tissue. (a) The anesthetized mouse is placed on its back and a horizontal incision is made below the xiphoid process (yellow lines showing the first ribs joined to the sternum and xiphoid process below) and widened to about $1 \mathrm{~cm}$. (b) The muscle layer is incised to expose the liver lobe, and a slight pressure is exerted on the abdomen so that the edge of the lobe emerges (see inset). (c) A few drops of Superglue (white circles) are deposited on the skin around the emerged lobe and a coverslip is gently pressed over the lobe.
13| Test whether the lasers are functioning correctly.

I CAUTION Laser beams can cause serious eye damage.

14| Install the platform on the microscope and cover with the thermal blanket to heat the platform before placing the mouse on it.

\section{Mouse operation}

15| Anesthetize a mouse ( $20 \mathrm{~g})$ by i.p. injection of $100 \mu \mathrm{l}$ of anesthetic. Wait about 10 min for the mouse to be in a prolonged, deep state of unconsciousness.

\section{? TROUBLESHOOTING}

16| Disinfect the abdomen with $70 \%$ ethanol (a solution of $70 \%$ ethanol $/ 1 \%$ betadiene can also be used). This also avoids any loose hairs from entering the incision (Note: the animal's abdomen can be shaved).

17| Make a horizontal incision in the mid-abdomen, around $0.5 \mathrm{~cm}$ below the xiphoid process. Enlarge the incision to about $1 \mathrm{~cm}$ wide and dissociate the conjunctive from the skin and pull the skin away from the incision. If the incision has

been done correctly, the liver should be visible under the muscle layers. The incision should be higher than the edge of the liver lobe. Remove any hairs from the incision with $1 \times$ PBS and immediately cauterize any blood vessels that have been cut with the cautery (Fig. 1a).

$\triangle$ CRITICAL STEP It is important to cauterize blood vessels as quickly as possible, as any coagulated blood will form a layer between the abdomen and the cover glass that will impede good imaging conditions.

? TROUBLESHOOTING

18| Make an incision in the muscle layer. Clean with $1 \times$ PBS. Put slight pressure on the abdomen to allow the edge of the left lobe to emerge. If the incision is in the correct place, the edge of the lobe will remain at the exterior (Fig. 1b). Note that the liver lobe does not necessarily need to be emerged to be observed. The cover glass can be glued over the visible zone of the exposed lobe, as described in Step 19, after the incision in the muscle layer.

19| Place a drop of $1 \times$ PBS on the liver lobe so that the tissue will not dehydrate during the imaging procedure. Place 5-6 small drops of Superglue 3 on the skin around the incision. Place a $24 \times 60 \mathrm{~mm}$ cover glass over the liver lobe and gently press down, sticking the cover glass in place (Fig. 1c).

20| Place the animal on the heated platform, and accommodate the center of the cover glass, and thus the liver lobe with the hole of the platform. Gently cover the animal with the thermal blanket (Fig. 2).

$\triangle$ CRITICAL STEP To avoid drying out of the animal's eyes, place cotton pads saturated in physiological serum over both eyes.

21 Switch on the mercury arc lamp, and using the filters for red fluorescence, turn the light path toward the ocular and search for the hepatic tissue using the autofluorescence of hepatocytes.

$\triangle$ CRITICAL STEP Avoid long exposure to the mercury arc lamp, which can cause damage and photobleaching of the sample.

22| Without moving the mouse, inject $50 \mu \mathrm{l}$ of the solution containing sporozoites (optimal $\sim 10^{6}$ sporozoites) and fluorescent BSA into the retro-orbital sinus $(50 \mu \mathrm{g})$. To do this, place your hand gently on the mouse and draw the fur on the animal's head into the palm of your hand. This causes the

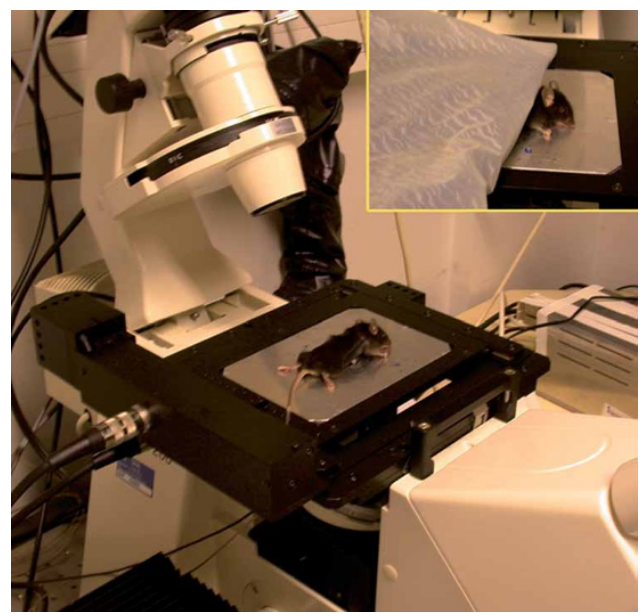

Figure 2 | Microscope setup for imaging the liver lobe. The animal is placed on the aluminum platform on the microscope stage as shown in the image. The lobe is placed so that it is in the center of the opening on the platform. In the inset, the mouse has been carefully covered with the homeothermic blanket to maintain its body temperature during image acquisition. 
animal's eye to proptose (bulge) slightly. The needle point is placed in the medial canthus (junction of eyelids closest to the animal's nose) and directed to a point directly behind the globe. Sufficient pressure must be applied to cut through the fibrous layer that surrounds the sinus. Inject the solution. The BSA will label the blood circulation, permitting the localization of liver sinusoids, and is a control of the injection for early stage visualization.

$\triangle$ CRITICAL STEP For later time-point studies (i.e., if following Step 10B), only inject fluorescent BSA.

23| Prepare for image acquisition: switch the excitation light path from the mercury arc to the laser source, change the emission light path from the ocular to the detector and make any other necessary changes.

\section{Acquiring the images}

24| To visualize the mouse liver sinusoids, start live imaging and activate the $647 \mathrm{~nm}$ laser. You should be able to visualize the hepatic sinusoids, and the parenchyma appears as a dark area (no autofluorescence of the parenchyma should be visible).

$\triangle$ CRITICAL STEP These steps (Steps 24-28) should be performed as fast as possible and are just one of the several ways for adjusting the $z$-stack settings.

? TROUBLESHOOTING

25 The imaging is done through the intact liver capsule. Find and focus on the capsule and set this $z$ as the baseline. For our equipment: in the "Hardware Controller" window, click on the cursor in the "Move" panel and position the $z$-step focus controller at $80 \mu \mathrm{m}$.

26| Focus by switching back to the epifluorescence mode, searching for fluorescent sporozoites/EEFs and defining the observation field such that there are a maximum of events in one field.

27| Once the parasites of interest have been chosen, switch back to the laser mode and define the $z$-stack. For our equipment: the depth of the focal plane is now controlled using the $z$-step controller. Using the $z$-step focus controller (positioned at $80 \mu \mathrm{m}$ ), move the focus toward the $0 \mu \mathrm{m}$ position. When the first (most superficial) parasite appears, set the $z$-stack bottom limit to at least $5 \mu \mathrm{m}$ before it is visible. Continue moving toward the $0 \mu \mathrm{m}$ position. When the deepest parasite is out of focus, set the top limit of the $z$-stack.

$\triangle$ CRITICAL STEP It is better to avoid imaging the capsule of the liver lobe that is highly fluorescent and will overshadow the hepatic morphology observed at lower positions in a merged $z$-stack. If the capsule is acquired, the $z$-slice(s) can be removed during image analysis.

28| Adjust the exposure time and laser power for excitation at 488 and $647 \mathrm{~nm}$.

$\triangle$ CRITICAL STEP The time of exposure and the binning should be adjusted so that a reasonable noise-to-signal ratio is obtained with an exposure time as low as possible to keep the time between two consecutive time points as short as possible. For the binning, at $2 \times 2$ instead of $1 \times 1$, the spatial resolution will be worse, but the luminosity is better, so the exposure time is shorter. The image at a binning of $2 \times 2$ should be of sufficient quality, and the data files are four times smaller.

! CAUTION To minimize damage by photobleaching, a balance must be found between laser power and exposure time.

? TROUBLESHOOTING

\section{Recording movies}

29| Once the $z$-stack has been defined, set the length of recording and start recording. The value of this parameter depends on the objective of the experiment. For instance, to track sporozoites using our equipment: in the "Sequence Control" window, go to "Length (s)" option and set the recording time (we usually record movies of $600 \mathrm{~s}$ ). The "Experiment" window should show the real-time acquisition.

$\triangle$ CRITICAL STEP Check if all parameters (laser, z-step, time of exposure) are properly set looking at the real-time images. If not, stop the acquisition; correct the parameter(s) and re-start recording.

30| When the time of acquisition is completed, check the quality of the recorded images. If the recorded images are of good quality (the parameters chosen are optimal) and the parasites are still visible with the same position values, another time lapse can be recorded. For our equipment: in the "Sequence Control" window, repeat Step 29 until the desired length of time is recorded. ? TROUBLESHOOTING

31 At the end of the recording session, kill the mouse by dislocation of the neck, or according to national and institutional regulations.

\section{Exporting the images}

32| Export the images in an appropriate format for subsequent analysis. If the software being used does not allow for image analysis or you prefer to use different software for analysis, it is best to export images as .tif files, as different image acquisition software can generate incompatible end files.

$\square$ PAUSE POINT The stored images can be analyzed immediately or later. 
PROTOCOL

\section{TIMING}

Steps 1 and 2, infection of mosquitoes and development of sporozoites: 18-22 days

Steps 3-9, dissecting infected mosquitoes: $30 \mathrm{~min}-1 \mathrm{~h}$

Steps 10-14, experiment preparation: $\sim 30 \mathrm{~min}$

Steps 15-21, preparation of the animal: maximum $30 \mathrm{~min}$

Steps 22-28, blood flow labeling and microscope settings: a few minutes

Steps 29 and 30, time of recording: 30 min-2 $\mathrm{h}$ (depends on the experiment)

Step 32, export time (if necessary): $\sim 30$ min (depends on the images taken)

Add at least $45 \mathrm{~h}$ between Steps 9 and 10 if studying late stages

\section{? TROUBLESHOOTING}

Troubleshooting advice can be found in Table 1.

TABLE 1 | Troubleshooting table.

\begin{tabular}{llll}
\hline Step & Problem & Possible reason & Solution \\
\hline 9 & There are not enough sporozoites & Low infectivity & $\begin{array}{l}\text { If infectivity is low, the reaction of the sporozoites } \\
\text { in the tissue may not be optimal. Verify that the } \\
\text { sporozoites are able to glide. If gliding is positive, then } \\
\text { a suspension of } 500,000 \text { sporozoites can be injected }\end{array}$ \\
15 & The mouse is hyperventilating & $\begin{array}{l}\text { Each mouse reacts differently to the } \\
\text { anesthesia }\end{array}$ & $\begin{array}{l}\text { For imaging, it is important that the mouse is in a } \\
\text { deep state of unconsciousness; if the mouse is } \\
\text { hyperventilating and this does not seem to calm down, } \\
\text { then anesthetize another mouse and let the precedent } \\
\text { mouse recover }\end{array}$
\end{tabular}

17 The liver lobe is not visible The incision was made too low

If possible, try to enlarge the opening toward the xiphoid process (sternum); however, the incision should not be too large, as the lobe will not remain emerged

24 Image is not appearing in the "Experiment" window

28

Computer out of memory

Files too big
Camera is not working

Laser is not working

Wrong light pathway

For our equipment: software in view mode
Insufficient anesthetic
Check camera

Check if laser (for our equipment: if the channel A/B) is activated

Make sure the light pathway is directed toward the camera

Activate "Live Image"

Using the Hamamatsu camera (full chip) at binning $2 \times 2,672 \times 512$ pixel, each image is approximately $340 \mathrm{~kb}$. In generating 600 -s-long movies, recording five $z$-stacks in $488 \mathrm{~nm}$ and five $z$-stacks in $647 \mathrm{~nm}$ with an exposure time of $400 \mathrm{~ms}$, the computer generates 1,500 pictures, corresponding to approximately $500 \mathrm{Mb}$. If a binning of $1 \times 1$ is used, the files are four times bigger, which can lead to memory problems for image acquisition and later on image analysis

More anesthetic ( $25 \mu \mathrm{l})$ can be injected subcutaneously in between two acquisitions. If the animal starts to wake up during an acquisition, stop the recording and inject the anesthetic without moving the animal. Restart recording as soon as possible

\section{ANTICIPATED RESULTS}

The output of imaging can take various forms; the most common is a video made by projecting the $z$ information into a single image (frequently using a method called maximum intensity projection). The position in the $z$-axis is no longer known, so two cells may seem to be interacting, when in fact they are in two different $z$-positions. One way to avoid misinterpretation is to 
apply a color code to the images, based on the $z$-position. For instance, cells at a depth of 60-50 $\mu \mathrm{m}$ are colored in blue, 50-40 $\mu \mathrm{m}$ in red and so on. The output can also be presented as a series of images representing the $z$-stack or the time series. Sporozoites are highly motile cells; their trajectory and speed in different environments can be analyzed using the MultiTracker plugin in ImageJ. One example is given in Figure 3 and Supplementary Video 1 online. The trajectory of released merosomes can also be analyzed in this way. During later-stage studies, the analysis of the volume of merosome buds permits the estimation of how many merozoites are released per merosome. To do this, threshold the $z$-stacks and measure the parasite area in each stack. The area multiplied by the $z$-step gives a volume in pixels. This can then be transformed into micrometers cubed. The conversion of pixels into micrometers depends in part on the objective, the camera and the binning used, and these parameters must be taken into account. The study of the volume of schizonts and merosome buds could be criteria to analyze the development and infectivity of EEFs.

This protocol for imaging malaria parasites in the liver of rodents is based on the use of a high-speed spinning disk confocal microscope, which allows rapid acquisition without tissue damage. It permits the acquisition of images in four dimensions (volume + time) and thus to study the dynamic behavior of fluorescent Plasmodium parasites in the liver. The sporozoite, freshly inoculated by a mosquito, first glides in the liver sinusoids, then leaves the blood compartment by crossing the endothelial barrier and reaching the liver parenchyma, further traverses several hepatocytes and finally invades a final hepatocyte inside a parasitophorous vacuole, where the parasite can differentiate. Parasite differentiation lasts $\sim 50 \mathrm{~h}$ in vivo, consists of the formation of thousands of daughter cells inside the infected hepatocyte and culminates with their release into the blood via the formation and movement of merosomes. The protocol described here should be useful to better describe, quantitatively, each of these steps.

Examples of images acquired using this protocol are shown in Figures 3-5. Figures $\mathbf{3}$ and $\mathbf{4}$ document sporozoite gliding inside the liver. Figure $3 \mathrm{a}$ shows the movement of sporozoites recorded in the left lobe of the liver; the panel shows pseudocolored maximum projections of confocal images (red: 5-25 min; green: 28-50 min; blue: 50-60 min). Figure $\mathbf{3 b}$ shows the
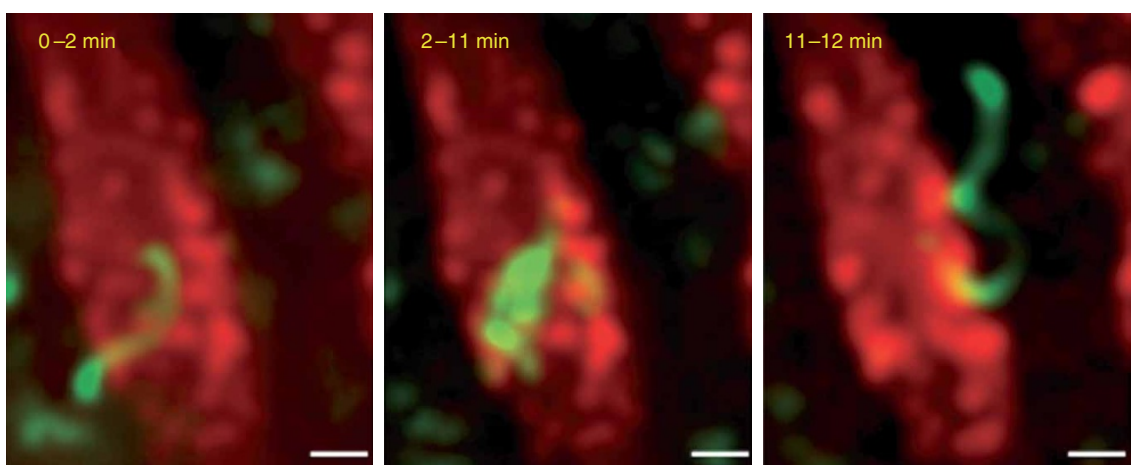

Figure 4 | Sporozoites in the sinusoids or the parenchyma of the liver (also see Supplementary Video 2 online). A sporozoite glides from the parenchyma into the sinusoid and back again into the parenchyma. The projected sporozoite movement for the time periods indicated on each panel is shown in yellow. The sinusoids (red) are labeled after intravenous injection of fluorescent BSA, which concentrates in cells exposed to the blood circulation, and the parenchyma corresponds to the black space between sinusoids. Scale bar, $10 \mu \mathrm{m}$. velocity profile (left) as well as displacement (right) of a single sporozoite that glides in the liver for more than $300 \mu \mathrm{m}$ before finally arresting. Figure 4 shows a time-lapse representation of a sporozoite gliding from the parenchyma into the sinusoid and back again into the parenchyma (see also Supplementary Video 2 online). Figure 5 and Supplementary Video 3 online show the release of merosomes from a mature exo-erythrocytic stage.

Such imaging approaches should lead to a better understanding of the primary infectious process of $P$. berghei/P. yoelii sporozoites in laboratory rodents. In addition, visualizing wild-type or mutant parasites in wild-type or mutant mice 

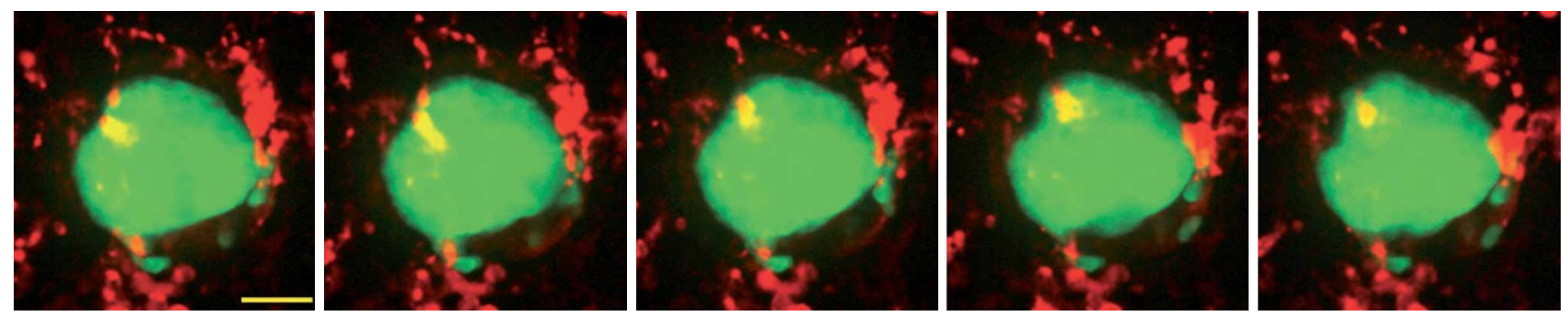

Figure 5 | Release of merosome buds from a hepatic schizont (also see Supplementary Video 3 online). Forty-eight hours after intravenous infection with sporozoites, the GFP-expressing parasites (green) are visualized in the hepatic tissue of a C57BL/6 mouse. The liver sinusoids are visualized using intravenously injected fluorescent BSA (red), and the hepatic parenchyma is visualized as a dark region. The images are composed of a $z$-projection of three $z$-stacks (covering $4 \mu \mathrm{m})$. Scale bar, $15 \mu \mathrm{m}$.

should also help in revealing the molecular basis of infection. Real-time in vivo imaging in the liver should also be valuable for visualizing anti-parasite immunity in context. The development of many mouse strains that produce fluorescent cell lines, such as myelomonocytic cells ${ }^{21}$, dendritic cells ${ }^{22}$ or natural killer cells ${ }^{23}$, will help to dissect the role of these cell types during parasitic infection. Similar approaches should also permit illumination of how protective immunity is achieved and how activated T cells interact with infected hepatocytes, or comparison of immune effectors against parasites in naïve or vaccinated animals. Ultimately, similar techniques might also be applied to study other host-parasite combinations, such as rodent parasites in their natural host (e.g., P. berghei-Grammomys surdaster) or primate systems, or $P$. falciparum in humanized mice grafted with human cells or tissues ${ }^{24}$.

Note: Supplementary information is available via the HTML version of this article.

ACKNOWLEDGMENTS We thank G. Milon, F. Frischknecht and P. Brey for experimental advice; A. Genovesio, C. Zimmer and J.C. Olivo-Marin for helping with tracking analysis; E. Perret, P. Roux, M. Marchand and C. Machu for helping with imaging; C. Bourgouin, I. Thiéry and the other members of the Center for Production and Infection of Anopheles for rearing the mosquitoes; and B. FrankeFayard, A. Waters and C. Janse for providing the PbGFP ${ }_{\text {CON }}$ parasite. This work was supported by funds from the Pasteur Institute (Strategic project "Grand Programme Horizontal Anopheles"), the Howard Hughes Medical Institute and the European Commission (FP6 BioMalPar Network of Excellence). S.B. is financed by a Roux fellowship (Institut Pasteur).

COMPETING INTERESTS STATEMENT The authors declare no competing financial interests.

Published online at http://www.natureprotocols.com

Reprints and permissions information is available online at http://npg.nature.com/ reprintsandpermissions

1. Boyd, M.F. \& Kitchen, S.F. The demonstration of sporozoites in human tissues. Am. J. Trop. Med. 9, 27-31 (1939).

2. Ponnudurai, T., Lensen, A.H., van Gemert, G.J., Bolmer, M.G. \& Meuwissen, J.H. Feeding behaviour and sporozoite ejection by infected Anopheles stephensi. Trans. R. Soc. Trop. Med. Hyg. 85, 175-180 (1991).

3. Sidjanski, S. \& Vanderberg, J.P. Delayed migration of Plasmodium sporozoites from the mosquito bite site to the blood. Am. J. Trop. Med. Hyg. 57, 426-429 (1997).

4. Vanderberg, J.P. \& Frevert, U. Intravital microscopy demonstrating antibodymediated immobilisation of Plasmodium berghei sporozoites injected into the skin by mosquitoes. Int. J. Parasitol. 9, 991-996 (2004).

5. Amino, R. et al. Quantitative imaging of Plasmodium transmission from mosquito to mammal. Nat. Med. 12, 220-224 (2006).

6. Prudencio, M., Rodriguez, A. \& Mota, M.M. The silent path to thousands of merozoites: the Plasmodium liver stage. Nat. Rev. Microbiol. 11, 849-856 (2006).

7. Meis, J.F., Verhave, J.P., Jap, P.H. \& Meuwissen, J.H. An ultrastructural study on the role of Kupffer cells in the process of infection by Plasmodium berghei sporozoites in rats. Parasitology 86, 231-242 (1983).
8. Meis, J.F. \& Verhave, J.P. Exoerythrocytic development of malarial parasites. Adv. Parasitol. 27, 1-61 (1988).

9. Pradel, G. \& Frevert, U. Malaria sporozoites actively enter and pass through rat Kupffer cells prior to hepatocyte invasion. Hepatology 33, 1154-1165 (2001).

10. Paddock, S.W. It was twenty years ago today: a celebration of optical sectioning. Biotechniques 35, 1156-1162 (2003).

11. Hell, S.W. Toward fluorescence nanoscopy. Nat. Biotechnol. 21, 1347-1355 (2003).

12. White, N. \& Errington, R. Multi-photon microscopy: seeing more by imaging less. Biotechniques 33, 298-305 (2002).

13. Nakano, A. Spinning-disk confocal microscopy: a cutting-edge tool for imaging of membrane traffic. Cell Struct. Funct. 27, 349-355 (2002).

14. Natarajan, R. et al. Fluorescent Plasmodium berghei sporozoites and preerythrocytic stages: a new tool to study mosquito and mammalian host interactions with malaria parasites. Cell Microbiol. 3, 371-379 (2001).

15. Franke-Fayard, B. et al. A Plasmodium berghei reference line that constitutively expresses GFP at a high level throughout the complete life cycle. Mol. Biochem. Parasitol. 137, 23-33 (2004).

16. Tarun, A.S. et al. Quantitative isolation and in vivo imaging of malaria parasite liver stages. Int. J. Parasitol. 36, 1283-1293 (2006).

17. Frevert, U. et al. Intravital observation of Plasmodium berghei sporozoite infection of the liver. PLoS Biol. 3, e192 (2005).

18. Sturm, A. et al. Manipulation of host hepatocytes by the malaria parasite for delivery into liver sinusoids. Science 313, 1287-1290 (2006).

19. Ishino, T., Orito, Y., Chinzei, Y. \& Yuda, M. A calcium-dependent protein kinase regulates Plasmodium ookinete access to the midgut epithelial cell. Mol. Microbiol. 59, 1175-1184 (2006).

20. Sinden, R.E. et al. Maintenance of the Plasmodium berghei life cycle. Methods Mol. Med. 72, 25-40 (2002)

21. Faust, N., Varas, F., Kelly, L.M., Heck, S. \& Graf, T. Insertion of enhanced green fluorescent protein into the lysozyme gene creates mice with green fluorescent granulocytes and macrophages. Blood 96, 719-726 (2000).

22. Lindquist, R.L. et al. Visualising dendritic cell networks in vivo. Nat. Immunol. 5, 1243-1250 (2004).

23. Stetson, D.B. et al. Constitutive cytokine mRNAs mark natural killer (NK) and NK T cells poised for rapid effector function. J. Exp. Med. 198, 1069-1076 (2003).

24. Melkus, M.W. et al. Humanized mice mount specific adaptive and innate immune responses to EBV and TSST-1. Nat. Med. 12, 1316-1322 (2006). 